\title{
OUTER VERSUS INNER HALO GLOBULAR CLUSTERS: NGC 7492 ABUNDANCES ${ }^{1}$
}

\author{
Judith G. Cohen ${ }^{2}$ and Jorge Melendez ${ }^{2}$ \\ Received 2004 October 18; accepted 2004 November 22
}

\begin{abstract}
We have carried out a detailed abundance analysis for 21 elements in a sample of four RGB stars in the outer halo globular cluster NGC $7492\left(R_{\mathrm{GC}} 25 \mathrm{kpc}\right)$; we find $[\mathrm{Fe} / \mathrm{H}]=-1.82$ dex inferred from $\mathrm{Fe}$ I lines $(-1.79$ from $\mathrm{Fe}$ II $)$ using high-dispersion $(R=\lambda / \Delta \lambda=35,000)$ spectra obtained with HIRES at the Keck Observatory. Most elements show no sign of star-to-star variation within our limited sample. We have, however, detected an anticorrelation between $\mathrm{O}$ and $\mathrm{Na}$ abundances similar to that seen in our previous analyses of inner halo GCs as well as in studies of relatively nearby GCs by others. We compare the abundance ratios in NGC 7492 with those we previously determined for the much closer old halo GCs M3 and M13. After making corrections for trends of abundance ratio with metallicity characteristic of halo stars, we find that for these three GCs, for each of the elements in common we deduce identical abundance ratios with respect to $\mathrm{Fe}$ to within the probable measurement uncertainties. Thus, the chemical history of the outer halo as exemplified by the metal-poor outer halo globular cluster NGC 7492 is indistinguishable from that of the inner halo, exemplified by M3 and M13, at least through the epoch of formation of these old globular clusters. This applies to the neutron capture processes as well.
\end{abstract}

Key words: Galaxy: halo — globular clusters: general — globular clusters: individual (NGC 7492) — stars: abundances

Online material: machine-readable table

\section{INTRODUCTION}

Abundance determinations of stars in Galactic globular clusters can provide valuable information about important astrophysical processes such as stellar evolution, stellar structure, Galactic chemical evolution, and the formation of the Milky Way. With the advent of efficient high-resolution spectrographs on 8-10 $\mathrm{m}$ telescopes, it is now possible to reach at least the luminous RGB stars in even the most distant Galactic globular clusters (GCs). In our previous work in this area we have explored the abundances for large samples of stars in the canonical relatively nearby GCs M71 (Ramírez et al. 2001; Ramírez \& Cohen 2002), M5 (Ramírez \& Cohen 2003), M3, and M13 (Cohen \& Melendez 2005), as well as in Pal 12 (Cohen 2004), a cluster associated with the Sgr dwarf galaxy (Irwin 1999; Dinescu et al. 2000; Ibata et al. 2001). For the case of Pal 12, in addition to the difference in age, it being several Gyr younger than the bulk of the Galactic GCs (Rosenberg et al. 1998, 1999), we found evidence for substantive differences between the chemical history of Pal 12 and that of the "normal" halo GCs of similar $[\mathrm{Fe} / \mathrm{H}]^{3}$

In the present work, we study the outer halo GC NGC 7492, a cluster that is not suspected (yet) of being part of any known stream or otherwise abnormal. We compare the abundance ratios deduced from high-resolution, high signal-to-noise ratio observations for four giants in this distant outer halo GC with those from our recent analysis of a large sample of stars in the relatively nearby and well-studied GCs M3 and M13, which have metallicities close to that of NGC 7492. We look for

\footnotetext{
${ }^{1}$ Based in part on observations obtained at the W. M. Keck Observatory, which is operated jointly by the California Institute of Technology, the University of California, and the National Aeronautics and Space Administration.

2 Palomar Observatory, MS 105-24, California Institute of Technology, Pasadena, CA 91125; jlc@astro.caltech.edu, jorge@astro.caltech.edu.

${ }^{3}$ The standard nomenclature is adopted; the abundance of element $\mathrm{X}$ is given by $\epsilon(\mathrm{X})=N(\mathrm{X}) / N(\mathrm{H})$ on a scale where $N(\mathrm{H})=10^{12} \mathrm{H}$ atoms. Then $[\mathrm{X} / \mathrm{H}]=$ $\log [N(\mathrm{X}) / N(\mathrm{H})]-\log [N(\mathrm{X}) / N(\mathrm{H})]_{\odot}$, and similarly for $[\mathrm{X} / \mathrm{Fe}]$.
}

evidence in the deduced abundance ratios of some difference in the formation mechanisms or chemical history of GCs in the "normal" outer halo.

There has been no previous high-dispersion study of NGC 7492. Zinn \& West (1984) obtained $[\mathrm{Fe} / \mathrm{H}]=-1.5 \pm 0.3$ dex using their narrow band Q39 photometric system, Smith (1984) derived $-1.34 \pm 0.25$ dex from the $\Delta S$ method applied to two RR Lyrae variables in the cluster, while moderate dispersion spectroscopy in the region of the infrared Ca triplet by Rutledge et al. (1997a) gave $[\mathrm{Fe} / \mathrm{H}]=-1.70 \pm 0.06 \mathrm{dex}$.

\section{STELLAR SAMPLE, OBSERVATIONS, AND $T_{\text {eff }}$ DETERMINATION}

Given the large distance of NGC 7492, we select the brightest possible stars on the upper RGB for observation, making no attempt to reach fainter luminosities. These stars were picked from the photometric study of this cluster by Cuffey (1961). Since this is a rather sparse cluster, these stars, which are the four brightest suspected members, lie distributed along the upper RGB, not concentrated at the RGB tip. The positions of these stars in a $V$ and $I \mathrm{CMD}$ are illustrated in Figure 1 superposed on the predicted cluster isochrone from the Yi et al. (2001) evolutionary tracks; see Figure 10 of Buonanno et al. (1987) for a $B$ and $V$ CMD of NGC 7492. Although the cluster is distant, there are so few luminous giants that they must be observed as single stars; two of them cannot fit into a single $7^{\prime \prime}$ long slit. Spectra were obtained with HIRES (Vogt et al. 1994) at the Keck Observatory on 20-22 August 2003. The instrument configuration covered the range 4650 to $7010 \AA$, with small gaps between the orders at the red end. This is the "yellow" configuration described in Cohen \& Melendez (2005). These data were reduced using a combination of Figaro (Shortridge 1993) scripts and the software package MAKEE. ${ }^{4}$

\footnotetext{
${ }^{4}$ MAKEE was developed by T. A. Barlow specifically for reduction of Keck HIRES data. It is freely available on the Web at the Keck Observatory home page, http://www2.keck.hawaii.edu/inst/hires/makeewww/index.html.
} 


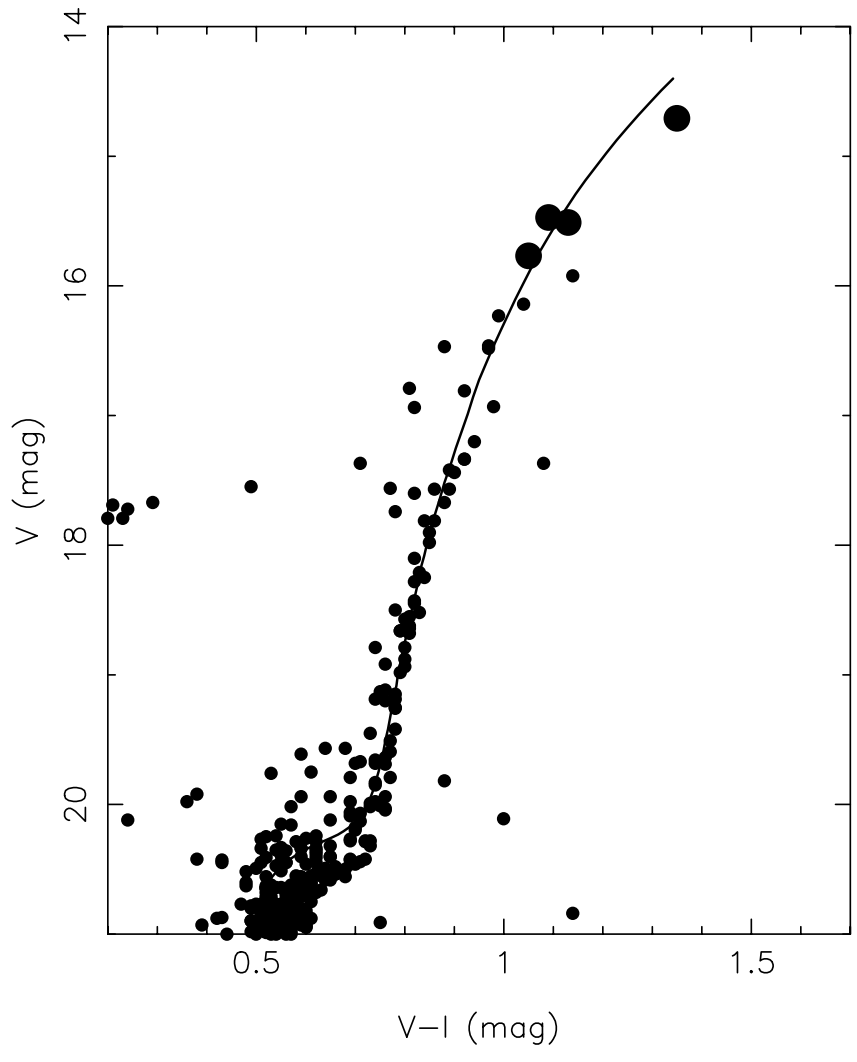

FIG. 1.-Plot of the $V-I$ CMD of NGC 7492: the four RGB stars observed with HIRES (large filled circles) are shown superposed on a $12 \mathrm{Gyr}$ isochrone from Yi et al. (2001) with $[\mathrm{Fe} / \mathrm{H}]-1.7$ dex shifted to the distance of NGC 7492. The small circles denote stars from Buonanno et al. (1987) in this GC roughly transformed from $B, V$ to $V, I$.

The desired minimum $\mathrm{S} / \mathrm{N}$ was 90 over a 4 pixel resolution element for a wavelength near the center of the HIRES detector. This is calculated strictly from the counts in the object spectrum, and excludes noise from cosmic ray hits, sky subtraction, flattening problems, etc. Since the nights were relatively dark, sky subtraction is not an issue except at the specific wavelengths corresponding to strong night sky emission lines, such as the $\mathrm{Na} \mathrm{D}$ doublet. The seeing was extremely good during these nights, making the exposures shorter than normal, and enabling us to reach this goal for the stars reported here. Table 1 gives details of the HIRES exposures for each star, with the total exposure time for each object. All long integrations were broken up into separate exposures, each $1200 \mathrm{~s}$ long, to optimize cosmic ray removal. The last column of the table gives the heliocentric radial velocity for each star, measured from the HIRES spectra; see Ramírez \& Cohen (2003) for details of the procedure used to determine $v_{r}$.

The radial velocity of NGC 7492 is large and negative, and the cluster abundance is low. It was easy to tell after one integration whether or not a star is a member of the cluster. Approximate measurements of $v_{r}$ were made on line; all stars attempted turned out to be radial velocity members of NGC 7492. The four RGB stars have a mean $v_{r}$ of $-176.9 \mathrm{~km} \mathrm{~s}^{-1}$, agreeing well within the errors with the early measurement of Hartwick \& Sargent (1978) of a single bright cluster member, but somewhat larger than the value of $-214 \mathrm{~km} \mathrm{~s}^{-1}$ found from moderateresolution spectra by Rutledge et al. (1997b). Our more accurate $v_{r}$ for NGC 7492 should be used in future computations of the mass of the Galaxy that rely on the orbits of its outlying satellites. The velocity dispersion from the four members, with no correction for an instrumental contribution, is $\sigma=1.2 \pm 1 \mathrm{~km} \mathrm{~s}^{-1}$, reflecting the low mass of this sparse cluster; the observed $\sigma$ is only slightly larger than the expected instrumental uncertainties.

\subsection{Stellar Parameter Determination}

We follow the procedures developed in our earlier work on globular cluster stars and described in Cohen et al. (2001) to determine the stellar parameters for the four RGB stars in NGC 7492. $T_{\text {eff }}$ is derived by comparing reddening-corrected broadband colors with the predictions of grids of model atmospheres. Here we use the grid of predicted broadband colors and bolometric corrections of Houdashelt et al. (2000) based on the MARCS stellar atmosphere code of Gustafsson et al. (1975).

We normally utilize $V-I, V-J$, and $V-K$ colors to determine $T_{\text {eff }}$. The infrared colors were taken from 2MASS (Skrutskie et al. 1997; Cutri et al. 2003). Cuffey (1961) provided $P$ and $V$ photographic photometry over a large field including the entire cluster of NGC $7492 .{ }^{5}$ CCD photometry in $B$ and $V$ was presented by Buonanno et al. (1987), but their field is smaller than the cluster and the calibration of their photometry is not secure. Côte et al. (1991), in the course of a study of blue stragglers in this cluster, obtained $\mathrm{CCD} B$ and $V$ photometry for a large sample of stars, but the data tables were never published and have subsequently been lost (P. C. Côté 2004, private communication).

The two published photometric studies of NGC 7492 give $V$ mag that are inconsistent by several tenths of a mag; the differences are not just a simple offset. We therefore measured $V$ and $I$ for our sample stars from ANDICAM images taken for this purpose

\footnotetext{
${ }^{5}$ Barnes (1968), who searched for variable stars in NGC 7492, defined his photometric system using Cuffey's measurements.
}

TABLE 1

The SAMPLE of Stars IN NGC 7492

\begin{tabular}{|c|c|c|c|c|c|c|c|c|}
\hline $\mathrm{ID}^{\mathrm{a}}$ & $\begin{array}{c}\text { R.A. } \\
(\mathrm{J} 2000.0)\end{array}$ & $\begin{array}{c}\text { Decl. } \\
(\mathrm{J} 2000.0)\end{array}$ & $\begin{array}{c}V^{\mathrm{b}} \\
(\mathrm{mag})\end{array}$ & $\begin{array}{c}I^{\mathrm{b}} \\
(\mathrm{mag})\end{array}$ & $\begin{array}{c}\text { Observation } \\
\text { Date }\end{array}$ & $\begin{array}{l}\text { Exposure } \\
\text { Time } \\
\text { (s) }\end{array}$ & $\mathrm{S} / \mathrm{N}^{\mathrm{c}}$ & $\begin{array}{c}v_{r} \\
\left(\mathrm{~km} \mathrm{~s}^{-1}\right)\end{array}$ \\
\hline H, 231 & 230822.32 & -153743 & 14.71 & 13.36 & 2003 Aug 20 & 800 & 88 & -176.8 \\
\hline $\mathrm{T}, 458 \ldots \ldots \ldots \ldots \ldots \ldots$ & 230825.75 & -153710 & 15.50 & 14.41 & 2003 Aug 21 & 3600 & 92 & -178.5 \\
\hline 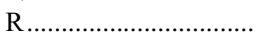 & 230829.46 & -153632 & 15.51 & 14.40 & 2003 Aug 20, 21 & 3000 & 92 & -175.5 \\
\hline $\mathrm{K}, 950 \ldots \ldots \ldots \ldots$ & 230820.83 & -153620 & 15.77 & 14.72 & 2003 Aug 22 & 3600 & 105 & -176.9 \\
\hline
\end{tabular}

NoтE.-Units of right ascension are hours, minutes, and seconds, and units of declination are degrees, arcminutes, and arcseconds.

a Alphabetical identifications are from Buonanno et al. (1987), and numerical ones are from Cuffey (1961).

${ }^{\mathrm{b}}$ Our photometry from ANDICAM images.

c Signal-to-noise ratio in the continuum near $5865 \AA$ A per 4 pixel spectral resolution element. 
TABLE 2

Stellar Parameters

\begin{tabular}{|c|c|c|c|}
\hline $\mathrm{ID}^{\mathrm{a}}$ & $\begin{array}{l}T_{\text {eff }} \\
(\mathrm{K})\end{array}$ & $\begin{array}{l}\log g \\
\text { (dex) }\end{array}$ & $\begin{array}{c}v_{t} \\
\left(\mathrm{~km} \mathrm{~s}^{-1}\right)\end{array}$ \\
\hline 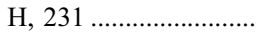 & 4300 & 0.62 & 2.0 \\
\hline R & 4650 & 1.18 & 2.0 \\
\hline 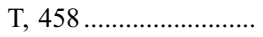 & 4715 & 1.21 & 2.0 \\
\hline $\mathrm{K}, 950 \ldots \ldots \ldots \ldots \ldots \ldots$ & 4750 & 1.33 & 2.0 \\
\hline
\end{tabular}

a Alphabetical identifications are from Buonanno et al. (1987), and numerical ones are from Cuffey (1961).

on 2004 August 1 with the $1.3 \mathrm{~m}$ telescope at CTIO operated by the SMARTS consortium; these values are given in Table 1. ANDICAM is a dual channel camera constructed by the Ohio State University instrument group, but only the optical channel was used. ${ }^{6}$ Our ANDICAM program requires photometric conditions, and additional standard star fields, charged to our ANDICAM allocation through NOAO, are always taken for us.

We adopt a distance for NGC 7492 of $26.2 \mathrm{kpc}$ (Côté et al. 1991) (as compared to $7.5 \mathrm{kpc}$ for M13 and $10.4 \mathrm{kpc}$ for M3), with a reddening of $E(B-V)=0.036$ mag from Schlegel et al. (1998). The relative extinction in various passbands is taken from Cohen et al. (1981; see also Schlegel et al. 1998). The adopted stellar parameters are given in Table 2 .

\subsection{Data Reduction and Analysis}

To the maximum extent possible, the atomic data and the analysis procedures used here are identical to those we used in our recently completed analysis of a large sample of stars in M3 and M13 (Cohen \& Melendez 2005). In particular, see $\S 3$ of that paper for a description of the measurement of the equivalent widths (listed for the four stars in NGC 7492 in Table 3), $\S 4$ for a discussion of the atomic parameters, $\S 4.2$ for our adopted solar abundances (tabulated in Table 2 of our earlier paper, and repeated as Table 4 here), and $\S 6$ for a description of our abundance analysis procedures. As in our earlier work, the abundance analysis is carried out using a current version of the LTE spectral synthesis program MOOG (Sneden 1973). We employ the grid of stellar atmospheres from Kurucz (1993) without convective overshoot, when available. The template file of suitable unblended lines with their adopted atomic parameters is identical to that we used in our recently completed analysis of M3 and of M13 (Cohen \& Melendez 2005).

Three of the four stars gave $v_{t}=2.0 \mathrm{~km} \mathrm{~s}^{-1}$ based on deriving a uniform $\mathrm{Fe}$ abundance as a function of $W_{\lambda}$ for the large

\footnotetext{
${ }^{6}$ See http://www.astronomy.ohio-state.edu/ANDICAM and http://www.astro .yale.edu/smarts.
}

TABLE 4

Adopted Solar Abundances

\begin{tabular}{|c|c|c|c|}
\hline Element & {$[\mathrm{X} / \mathrm{H}]^{\mathrm{a}}$} & Element & {$[\mathrm{X} / \mathrm{H}]^{\mathrm{a}}$} \\
\hline$\ldots \ldots \ldots$ & 8.85 & Fe...... & 7.45 \\
\hline $\mathrm{Na} . . . . . . . .$. & 6.33 & $\mathrm{Ni} . .$. & 6.25 \\
\hline 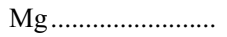 & 7.54 & 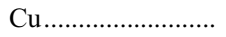 & 4.21 \\
\hline 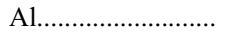 & 6.47 & $\mathrm{Zn}$ & 4.60 \\
\hline $\mathrm{Si}$ & 7.55 & $\mathrm{Ba} \ldots \ldots \ldots \ldots$ & 2.13 \\
\hline $\mathrm{Ca}$ & 6.36 & Y............. & 2.24 \\
\hline 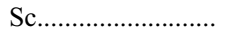 & 3.10 & $\mathrm{Zr} \ldots \ldots \ldots \ldots \ldots \ldots \ldots \ldots \ldots \ldots \ldots \ldots \ldots$ & 2.60 \\
\hline 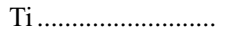 & 4.99 & $\mathrm{La}$ & 1.14 \\
\hline V & 4.00 & $\mathrm{Nd}$ & 1.45 \\
\hline 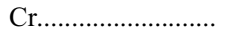 & 5.67 & Eu & 0.51 \\
\hline $\mathrm{Mn}$ & 5.39 & 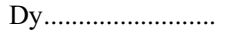 & 1.10 \\
\hline
\end{tabular}

${ }^{\text {a }}$ Given on a scale where $\log N(\mathrm{H})=12.0$; values in dex.

set of Fe I lines. The fourth star (star R) gave $1.8 \mathrm{~km} \mathrm{~s}^{-1}$; this value was also set to $2.0 \mathrm{~km} \mathrm{~s}^{-1}$. Lines with $W_{\lambda}>175 \mathrm{~m} \AA$ were ignored, except for the $\mathrm{Ba}$ II lines in the coolest star in our sample in NGC 7492.

The resulting abundance ratios for the four RGB stars in NGC 7492 are given in Tables 5A-5E. Species with only one detected line are assigned an uncertainty of 0.10 dex. Table 7 of Cohen \& Melendez (2005) indicates the changes in derived abundance ratios for small changes in the adopted stellar parameters, the $[\mathrm{Fe} / \mathrm{H}]$ for the adopted model atmosphere, or the set of $W_{\lambda}$ for the lines of each species, and is appropriate for use here as well. The mean abundance and $1 \sigma$ variance for the species observed in NGC 7492 are listed in Table 6.

\subsection{Comments on Individual Elements}

The oxygen abundance is derived from the forbidden lines at 6300 and $6363 \AA$. The subtraction of the night sky emission lines for the forbidden lines was reasonably straightforward given that the radial velocity of NGC 7492 is sufficiently different from $0 \mathrm{~km}$ $\mathrm{s}^{-1}$ that their $W_{\lambda}$ can be reliably measured. The $\mathrm{C} / \mathrm{O}$ ratio was assumed to be solar. $\mathrm{CN}$ and $\mathrm{Ni}$ I contamination is negligible (see Cohen \& Melendez 2005). [O/Fe] is given with respect to $\mathrm{Fe}$ II; abundance ratios for all other elements are given with respect to $\mathrm{Fe}$ I.

The deduced mean [Fe/H] of NGC 7492 value (see Fig. 2) is in good agreement with that inferred by Rutledge et al. (1997a) from moderate dispersion spectra in the region of the infrared Ca triplet.

The Na abundance was obtained from the $5680 \AA$ doublet for all four stars. As in our previous papers, we have not used any non-LTE corrections for Na. Calculations by Gratton et al. (1999) suggest values of between 0.1 and 0.2 dex are appropriate for our

TABLE 3

Equivalent Widths

\begin{tabular}{|c|c|c|c|c|c|c|c|}
\hline Ion & $\begin{array}{c}\lambda \\
(\AA)\end{array}$ & $\begin{array}{l}\chi_{\mathrm{exc}} \\
(\mathrm{eV})\end{array}$ & $\begin{array}{c}\log g f \\
(\operatorname{dex})\end{array}$ & $\begin{array}{c}231 \\
(\mathrm{~m} \AA)\end{array}$ & $\begin{array}{c}\mathrm{R} \\
(\mathrm{m} \AA)\end{array}$ & $\begin{array}{c}950 \\
(\mathrm{~m} \AA)\end{array}$ & $\begin{array}{c}458 \\
(\mathrm{~m} \AA)\end{array}$ \\
\hline \multirow[t]{2}{*}{ O I } & 6300.30 & 0.00 & -9.78 & 30.0 & 23.5 & 13.5 & 27.3 \\
\hline & 6363.78 & 0.02 & -10.30 & 9.0 & $\ldots$ & 8.0 & 9.5 \\
\hline \multirow[t]{3}{*}{ 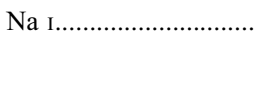 } & 5682.63 & 2.10 & -0.70 & 46.0 & 32.1 & 8.0 & 16.0 \\
\hline & 5688.19 & 2.10 & -0.42 & 61.0 & 49.6 & 15.0 & 28.5 \\
\hline & 6160.75 & 2.00 & -1.23 & 18.5 & $\ldots$ & $\ldots$ & $\ldots$ \\
\hline
\end{tabular}

Note.-Table 3 is published in its entirety in the electronic edition of the Astronomical Journal. A portion is shown here for guidance regarding its form and content. 
TABLE 5A

Abundance Ratios: O to Mg

\begin{tabular}{|c|c|c|c|c|c|c|c|c|c|c|}
\hline Star & $\begin{array}{c}{[\mathrm{Fe} / \mathrm{H}]_{\mathrm{I}}} \\
\pm \sigma / \sqrt{N} \\
\quad(\mathrm{dex})\end{array}$ & $N$ & $\begin{array}{c}{[\mathrm{Fe} / \mathrm{H}]_{\mathrm{II}}} \\
\pm \sigma / \sqrt{N} \\
\quad(\mathrm{dex})\end{array}$ & $N$ & $\begin{array}{c}{[\mathrm{O} / \mathrm{Fe}]} \\
\pm \sigma / \sqrt{N} \\
(\mathrm{dex})\end{array}$ & $N$ & $\begin{array}{c}{[\mathrm{Na} / \mathrm{Fe}]} \\
\pm \sigma / \sqrt{N} \\
(\mathrm{dex})\end{array}$ & $N$ & $\begin{array}{c}{[\mathrm{Mg} / \mathrm{Fe}]} \\
\pm \sigma / \sqrt{N} \\
(\mathrm{dex})\end{array}$ & $N$ \\
\hline $231 \ldots \ldots \ldots \ldots$ & $-1.76 \pm 0.05^{*}$ & 90 & $-1.72 \pm 0.06$ & 12 & $0.26 \pm 0.05^{*}$ & 2 & $0.10 \pm 0.05$ & 3 & $0.67 \pm 0.16$ & 3 \\
\hline 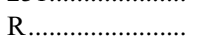 & $-1.77 \pm 0.05^{*}$ & 66 & $-1.80 \pm 0.08$ & 9 & $0.49 \pm 0.10$ & 1 & $0.19 \pm 0.05^{*}$ & 2 & $0.52 \pm 0.06$ & 3 \\
\hline 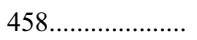 & $-1.79 \pm 0.05^{*}$ & 70 & $-1.77 \pm 0.05$ & 10 & $0.55 \pm 0.05^{*}$ & 2 & $-0.10 \pm 0.05^{*}$ & 2 & $0.55 \pm 0.20$ & 3 \\
\hline 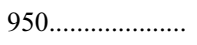 & $-1.94 \pm 0.05^{*}$ & 64 & $-1.88 \pm 0.05$ & 9 & $0.52 \pm 0.15$ & 2 & $-0.28 \pm 0.05^{*}$ & 2 & $0.45 \pm 0.20$ & 3 \\
\hline
\end{tabular}

Nоте.-Asterisk indicates that the minimum value of 0.05 dex has been adopted; the nominal calculated value is smaller.

TABLE 5B

Abundance Ratios: Si to V

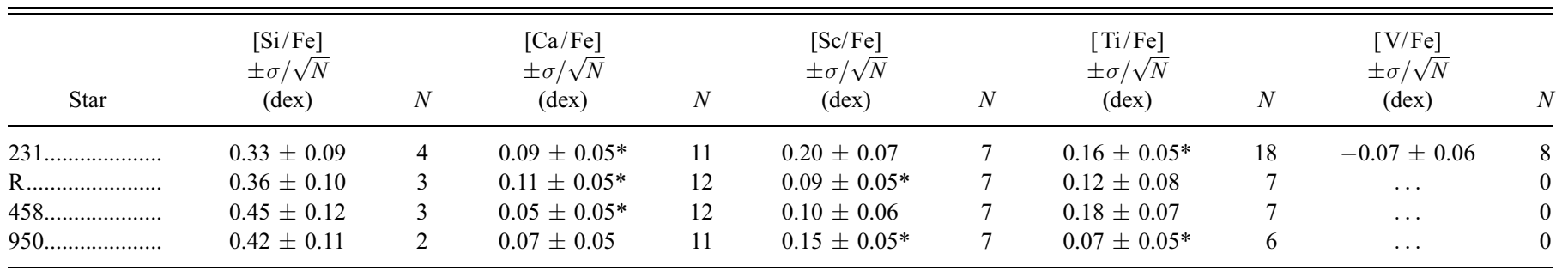

Nоте.-Asterisk indicates that the minimum value of 0.05 dex has been adopted; the nominal calculated value is smaller.

TABLE 5C

Abundance Ratios: $\mathrm{Cr}$ to $\mathrm{Cu}$

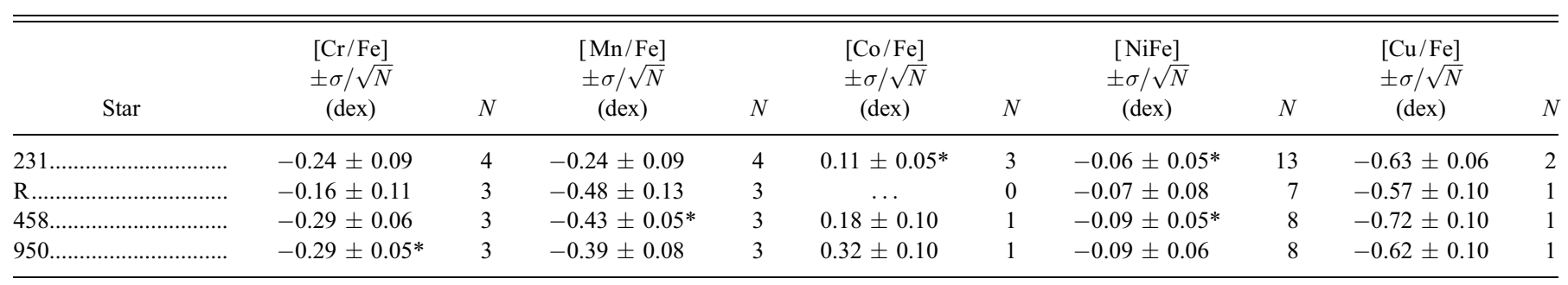

Noте.-Asterisk indicates that the minimum value of 0.05 dex has been adopted; the nominal calculated value is smaller.

TABLE 5D

Abundance Ratios: Zn to La

\begin{tabular}{|c|c|c|c|c|c|c|c|c|c|c|}
\hline Star & $\begin{array}{c}{[\mathrm{Zn} / \mathrm{Fe}]} \\
\pm \sigma / \sqrt{N} \\
(\mathrm{dex})\end{array}$ & $N$ & $\begin{array}{c}{[\mathrm{Y} / \mathrm{Fe}]} \\
\pm \sigma / \sqrt{N} \\
(\mathrm{dex})\end{array}$ & $N$ & $\begin{array}{c}{[\mathrm{Zr} / \mathrm{Fe}]} \\
\pm \sigma / \sqrt{N} \\
(\mathrm{dex})\end{array}$ & $N$ & $\begin{array}{c}{[\mathrm{Ba} / \mathrm{Fe}]} \\
\pm \sigma / \sqrt{N} \\
(\mathrm{dex})\end{array}$ & $N$ & $\begin{array}{c}{[\mathrm{La} / \mathrm{Fe}]} \\
\pm \sigma / \sqrt{N} \\
(\mathrm{dex})\end{array}$ & $N$ \\
\hline $231 \ldots \ldots$ & $-0.09 \pm 0.08$ & 2 & $-0.29 \pm 0.05^{*}$ & 4 & $0.36 \pm 0.10$ & 3 & $0.24 \pm 0.10$ & 3 & $0.12 \pm 0.16$ & 2 \\
\hline R & $-0.09 \pm 0.08$ & 2 & $-0.35 \pm 0.05^{*}$ & 4 & $\ldots$ & 0 & $0.05 \pm 0.09$ & 3 & $\ldots$ & 0 \\
\hline 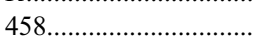 & $-0.16 \pm 0.05$ & 2 & $-0.31 \pm 0.06$ & 4 & $\ldots$ & 0 & $0.34 \pm 0.07$ & 3 & $\ldots$ & 0 \\
\hline 950 & $0.13 \pm 0.05^{*}$ & 2 & $-0.11 \pm 0.08$ & 4 & $\ldots$ & 0 & $0.50 \pm 0.08$ & 3 & $\ldots$ & 0 \\
\hline
\end{tabular}

Note.-Asterisk indicates that the minimum value of 0.05 dex has been adopted; the nominal calculated value is smaller. 
TABLE 5E

Abundance Ratios: Nd to Eu

\begin{tabular}{|c|c|c|c|c|}
\hline Star & $\begin{array}{c}{[\mathrm{Nd} / \mathrm{Fe}]} \\
\pm \sigma / \sqrt{N} \\
(\mathrm{dex})\end{array}$ & $N$ & $\begin{array}{c}{[\mathrm{Eu} / \mathrm{Fe}]} \\
\pm \sigma / \sqrt{N} \\
(\mathrm{dex})\end{array}$ & $N$ \\
\hline $231 \ldots \ldots \ldots \ldots \ldots \ldots$ & $0.40 \pm 0.05^{*}$ & 6 & $0.61 \pm 0.10$ & 1 \\
\hline R & $0.26 \pm 0.05^{*}$ & 4 & $\ldots$ & 0 \\
\hline $458 \ldots \ldots \ldots \ldots \ldots$ & $0.19 \pm 0.07$ & 4 & $\ldots$ & 0 \\
\hline 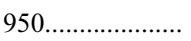 & $0.41 \pm 0.08$ & 4 & $\ldots$ & 0 \\
\hline
\end{tabular}

Nоте.-Asterisk denotes that the minimum value of 0.05 dex has been adopted; the nominal calculated value is smaller.

sample in NGC 7492, with the coolest star having the largest value. Calculations by Takeda et al. (2003) suggest somewhat smaller values.

The Ba abundance of star 950 in NGC 7492 appears to be $\sim 0.2$ dex larger than that of the other cluster members studied here. However, the detected lines of $\mathrm{Ba}$ II are all within the range where substantial hyperfine structure corrections occur. Table 7 of Cohen \& Melendez (2005) shows the very high sensitivity of the deduced $\mathrm{Ba}$ abundances to small uncertainties in the equivalent width and microturbulence of the $\mathrm{Ba}$ II lines we use. On the other hand, the deduced $[\mathrm{Y} / \mathrm{Fe}$ ] is also somewhat high for this star, while the deduced $\mathrm{Fe}$ is the lowest of all the stars in our sample. Perhaps a slight adjustment of $T_{\text {eff }}$ for this star is required. At this point, we assume this is the result of observational and modeling uncertainties and does not indicate a real spread in $[\mathrm{Ba} / \mathrm{Fe}]$ within NGC 7492 , but further verification of this is desirable.

The abundances of the elements with respect to $\mathrm{Fe},[\mathrm{X} / \mathrm{Fe}]$, as a function of $T_{\text {eff }}$ are shown in Figure 3 for $\mathrm{O}, \mathrm{Na}, \mathrm{Mg}$, and $\mathrm{Si}$;

TABLE 6

Mean Abundances and Spread Ratios

\begin{tabular}{|c|c|c|c|c|c|}
\hline Species & $\begin{array}{c}\text { Mean Abundance } \\
{[\mathrm{X} / \mathrm{Fe}]} \\
(\mathrm{dex})\end{array}$ & $\begin{array}{c}\sigma \\
(\operatorname{dex})\end{array}$ & $\begin{array}{c}\sigma(\text { tot }) \\
(\operatorname{dex})\end{array}$ & $\begin{array}{l}\text { Spread } \\
\text { Ratio }^{\text {a }} \\
(\text { dex })\end{array}$ & $\begin{array}{l}\text { No. of } \\
\text { Stars }\end{array}$ \\
\hline O I . & 0.46 & 0.13 & 0.10 & 1.30 & 4 \\
\hline 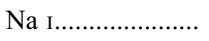 & -0.02 & 0.21 & 0.07 & 3.00 & 4 \\
\hline 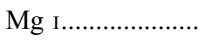 & 0.55 & 0.09 & 0.20 & 0.45 & 4 \\
\hline $\mathrm{Si}$ I $\ldots \ldots \ldots \ldots \ldots \ldots \ldots$ & 0.39 & 0.06 & 0.10 & 0.60 & 4 \\
\hline $\mathrm{Ca}$ I........................ & 0.08 & 0.03 & 0.12 & 0.25 & 4 \\
\hline Sc II ........................... & 0.14 & 0.05 & 0.21 & 0.24 & 4 \\
\hline Ті г........................ & 0.13 & 0.05 & 0.12 & 0.42 & 4 \\
\hline 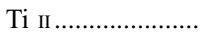 & 0.34 & 0.06 & 0.15 & 0.40 & 4 \\
\hline$V_{\text {I }} \ldots \ldots \ldots \ldots$ & -0.07 & $\ldots$ & 0.23 & $\ldots$ & 1 \\
\hline 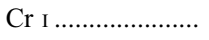 & -0.25 & 0.06 & 0.17 & 0.35 & 4 \\
\hline 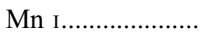 & -0.39 & 0.10 & 0.18 & 0.55 & 4 \\
\hline $\mathrm{Fe}$ I & -1.82 & 0.08 & 0.10 & 0.80 & 4 \\
\hline $\mathrm{Fe}$ II & -1.79 & 0.07 & 0.13 & 0.54 & 4 \\
\hline 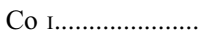 & 0.20 & 0.11 & 0.12 & 0.92 & 3 \\
\hline 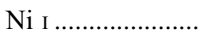 & -0.08 & 0.02 & 0.10 & 0.20 & 4 \\
\hline $\mathrm{Cu}$ I......................... & -0.64 & 0.06 & 0.16 & 0.38 & 4 \\
\hline 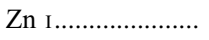 & -0.03 & 0.11 & 0.13 & 0.85 & 4 \\
\hline 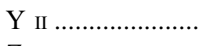 & -0.27 & 0.11 & 0.15 & 0.73 & 4 \\
\hline 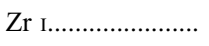 & 0.36 & $\ldots$ & 0.16 & $\ldots$ & 1 \\
\hline 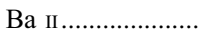 & 0.28 & 0.19 & 0.22 & 0.86 & 4 \\
\hline La II ....................... & 0.12 & $\ldots$ & 0.18 & $\ldots$ & 1 \\
\hline 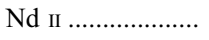 & 0.32 & 0.11 & 0.11 & 1.00 & 4 \\
\hline Eu II ......................... & 0.61 & $\ldots$ & $\ldots$ & $\ldots$ & 1 \\
\hline
\end{tabular}

${ }^{\text {a }}$ Ratio of $\sigma$ to $\sigma$ (tot). See text.

e The number of stars in which lines of this species were detected.

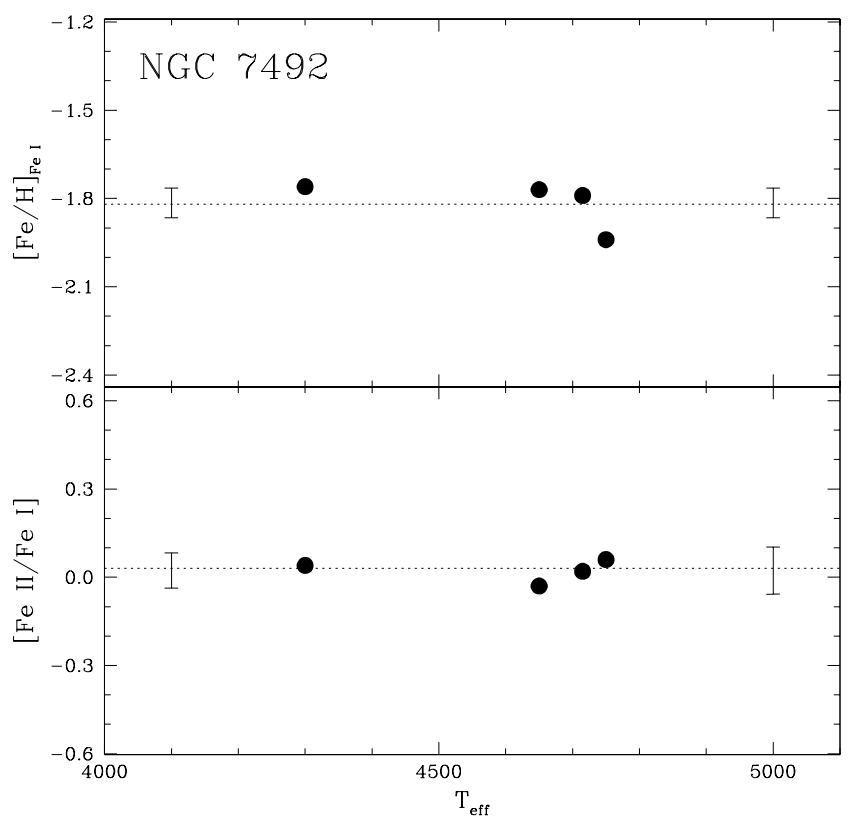

FIG. 2.- $[\mathrm{Fe} / \mathrm{H}]$ from lines of $\mathrm{Fe} \mathrm{I}$, shown as a function of $T_{\text {eff }}$ in the upper panel; lower panel shows the ionization equilibrium of $\mathrm{Fe}$ for our sample of four stars in NGC 7492. The error bars on the left are those of the most luminous star, while the error bars on the right are those of the faintest star in our sample. The dotted horizontal line indicates the mean value for our sample in this GC.

Figure 4 for $\mathrm{Ca}, \mathrm{Sc}$, Ti, and V; Figure 5 for $\mathrm{Cr}, \mathrm{Mn}, \mathrm{Co}$, and $\mathrm{Ni}$; Figure 6 for $\mathrm{Cu}, \mathrm{Zn}, \mathrm{Y}$, and Zr; and Figure 7 for $\mathrm{Ba}, \mathrm{La}, \mathrm{Nd}, \mathrm{Eu}$, and Dy. Note the apparent star-to-star variation in $[\mathrm{O} / \mathrm{Fe}]$ and in $[\mathrm{Na} / \mathrm{Fe}]$, which becomes undetectably small, if it exists at all, for the elements heavier than $\mathrm{Na}$. The scatter for $[\mathrm{Ca} / \mathrm{Fe}]$ and [Ni/Fe], with 8 to 13 detected absorption lines in each star, is remarkably small, $\leq 0.03$ dex over the four-star sample in NGC 7492.

\subsection{Abundance Spreads}

To check for the presence of star-to-star variations in abundance ratios within the small sample of RGB stars in NGC 7492,

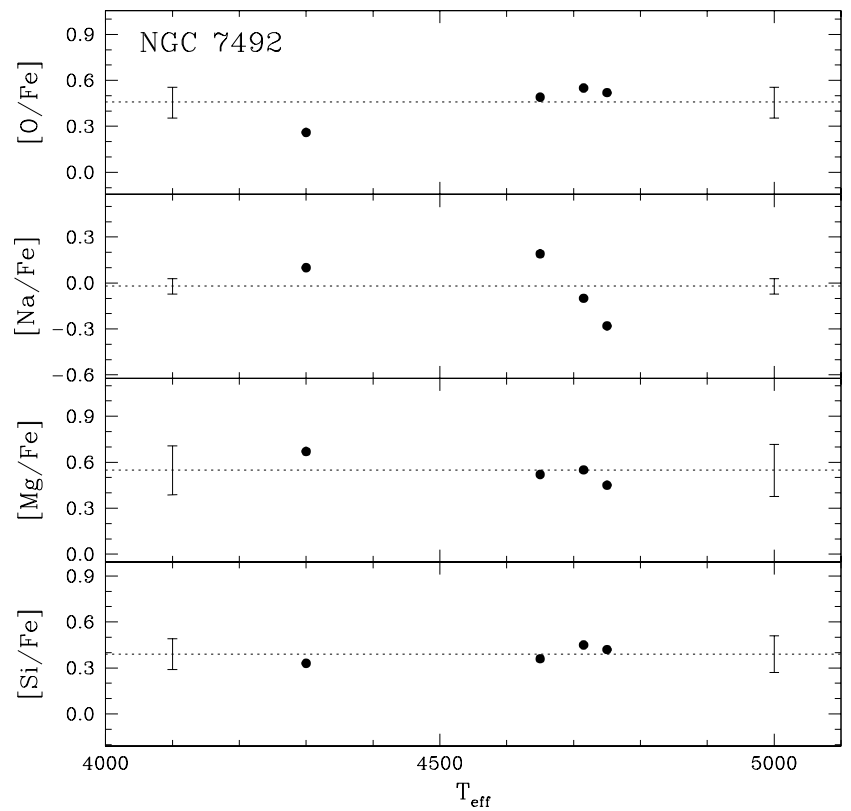

FIG. 3.- $[\mathrm{X} / \mathrm{Fe}]$ for the elements $\mathrm{O}, \mathrm{Na}, \mathrm{Mg}$, and $\mathrm{Si}$, shown as a function of $T_{\text {eff }}$ for our sample of four stars in NGC 7492. The error bars for the most luminous and least luminous stars, as well as the cluster mean, are as in Fig. 2. 


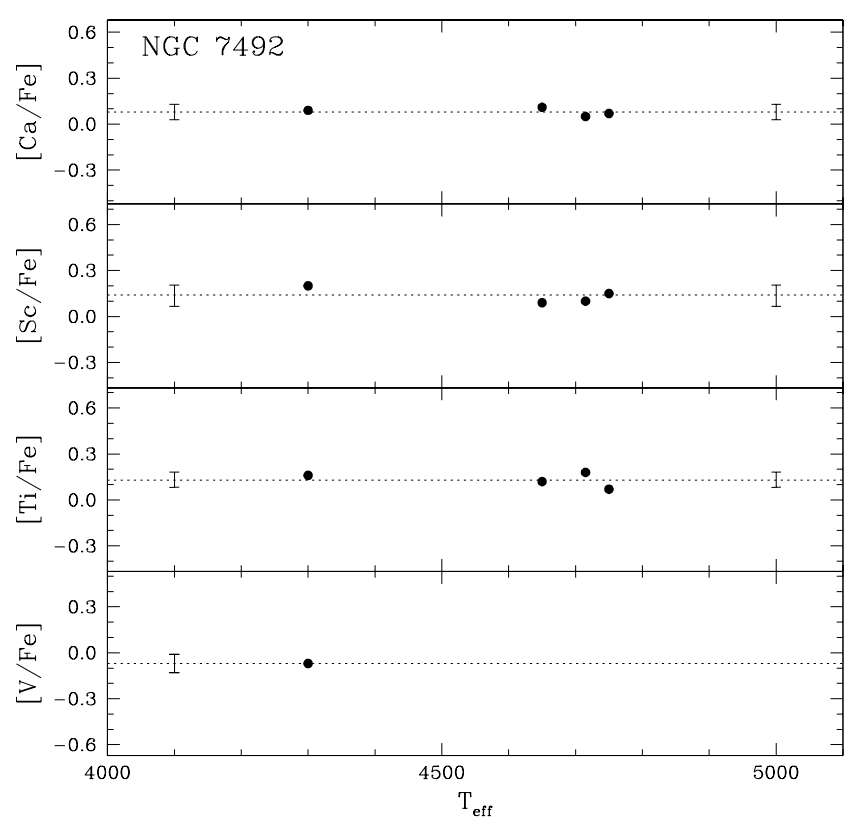

FIG. 4.- Same as Fig. 3, but for Ca, Sc, Ti, and V in NGC 7492.

we use a parameter we call the "spread ratio" (SR). The numerator of SR is the $1 \sigma$ rms variance for the sample of four stars in NGC 7492 about the mean abundance for each atomic species $(X)$ with detected absorption lines, denoted $\sigma$; the relevant values are given in the first three columns of Table 6 . The denominator of SR is the total expected uncertainty, $\sigma$ (tot), which is the sum in quadrature of the known contributing terms. Included are a term corresponding to an uncertainty of $50 \mathrm{~K}$ in $T_{\text {eff }}$, the same for an uncertainty of 0.2 dex in $\log g$, and for an uncertainty of $0.2 \mathrm{~km} \mathrm{~s}^{-1}$ in $v_{t}$, and the observed uncertainty $[\sigma(\mathrm{obs})]$. The parameter $\sigma(\mathrm{obs})$, which is calculated from data given in Tables $5 \mathrm{~A}-5 \mathrm{E}$, is taken as the variance about the mean abundance for a given species in a given star, i.e., the $1 \sigma \mathrm{rms}$ value about the mean abundance of species $X$ in a given star divided by $\sqrt{N}$, where $N$ is the number of observed lines of spe-

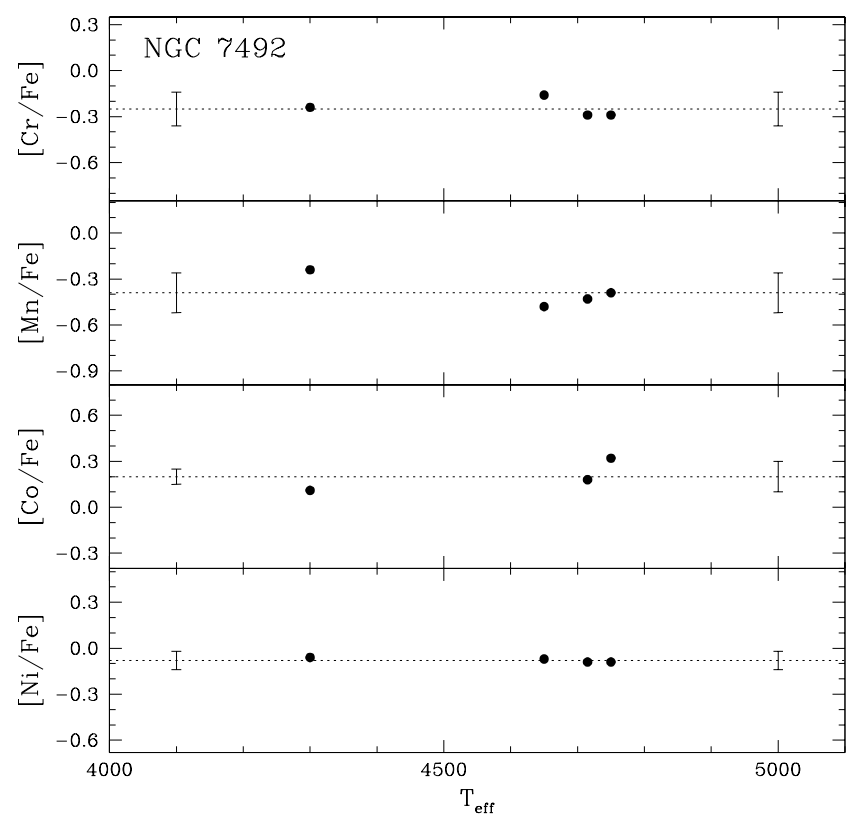

FIG. 5.- Same as Fig. 3, but for Cr, Mn, Co, and Ni in NGC 7492.

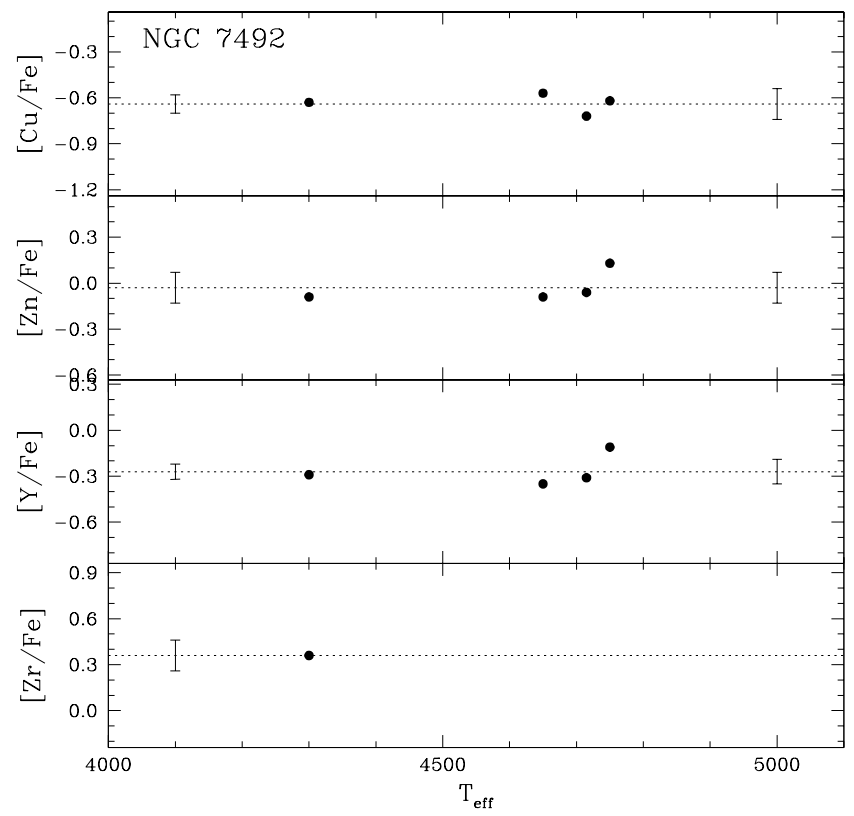

FIG. 6.- Same as Fig. 3, but for Cu, Zn, Y, and Zr in NGC 7492.

cies $X$. It includes contributions from errors in the measured $W_{\lambda}$, random errors (i.e., between lines of a given species) in the adopted $g f$ values, etc. Some species, for example Fe I, with its very large value of $N$, have unrealistically small values of $\sigma(\mathrm{obs})$; for these we adopt a minimum of 0.05 dex for this parameter.

The ratio $\sigma / \sigma$ (tot) is an indication of whether there is any intrinsic star-to-star variation in $[\mathrm{X} / \mathrm{Fe}]$. A high value of this $\mathrm{SR}$, tabulated in the fifth column of Table 6, suggests a high probability of intrinsic scatter for the abundance of the species $X$. Ideally, the mean SR for those elements with no star-to-star variation should be unity; for many species the measured SR is close to that value, certainly closer here than for the sample in M3 and in M13 we studied earlier (Cohen \& Melendez 2005).

Inspection of Table 6 shows that for all but two species SR $<$ 1.0 for the sample of four stars in NGC 7492, indicating little

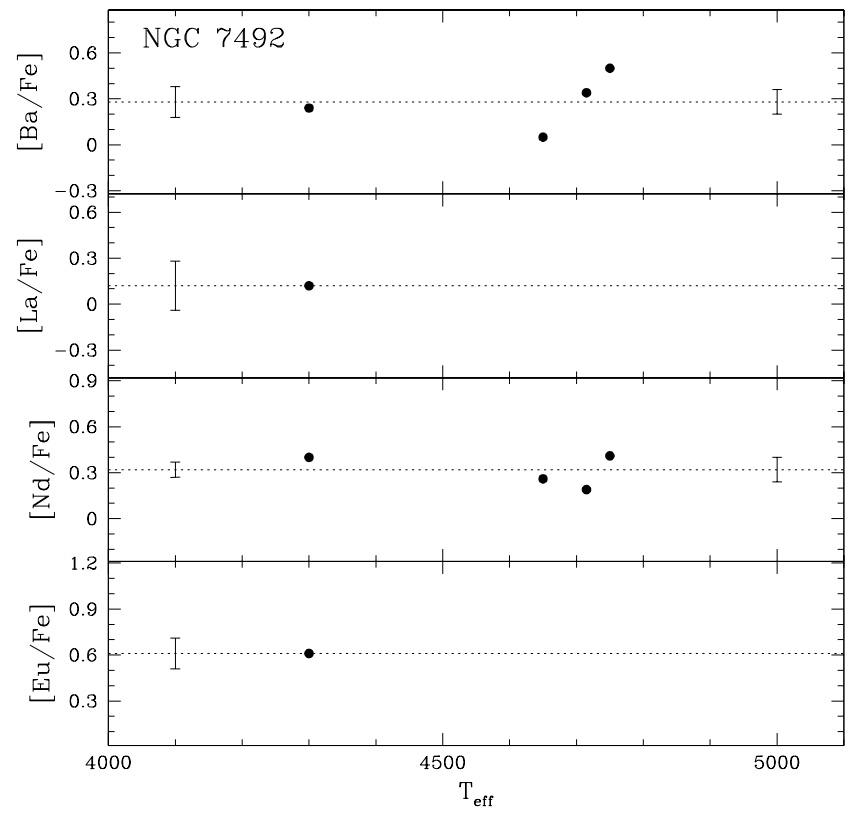

Fig. 7.- Same as Fig. 3, but for Ba, La, Nd, and Eu in NGC 7492. 


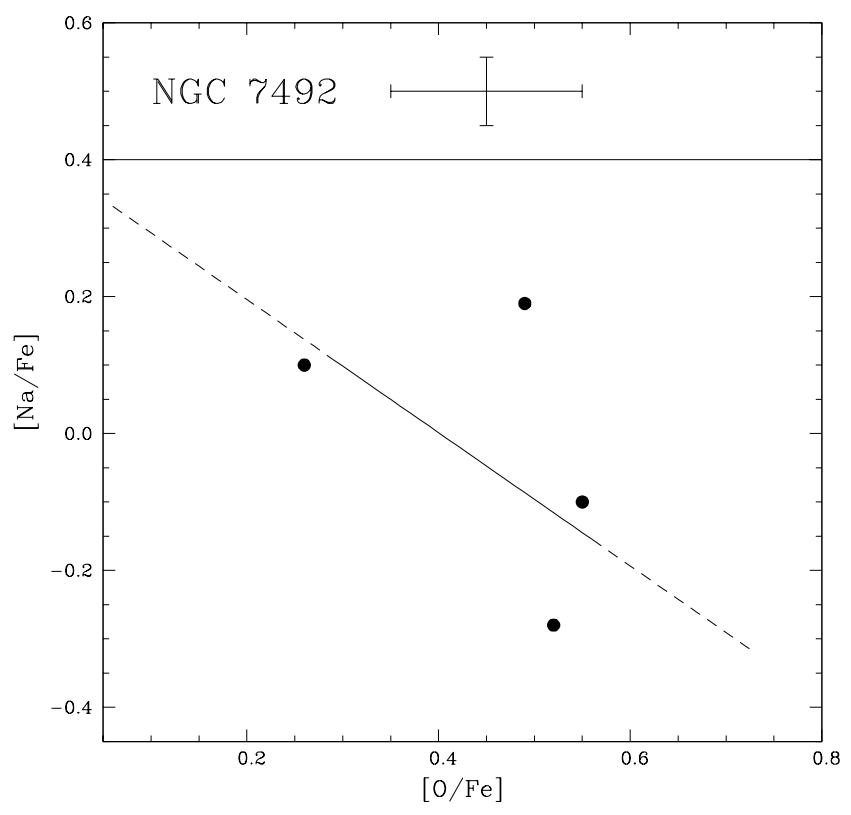

Fig. 8. - Ratio $[\mathrm{Na} / \mathrm{Fe}]$ shown as a function of $[\mathrm{O} / \mathrm{Fe}]$ for our sample of four stars in NGC 7492. The error bars typical of the most luminous and least luminous stars in our sample are indicated. The line represents the relationship found by Sneden et al. (2004), with a shift of -0.07 and +0.1 dex in the vertical and horizontal axis with respect to the relation we found for M13 (Cohen \& Melendez 2005); the line is solid between the first and third quartiles of Sneden et al. (2004) M3 sample and dashed outside that regime.

sign of an intrinsic star-to-star range in abundance. $\mathrm{O}$ I and $\mathrm{Na}$ I, however, have the two largest values of SR, 1.3 and 3.0, respectively. Note that SR for Mg I is 0.5 , suggesting no real star-tostar abundance variations for this element. We therefore assume that the range of abundances seen in our sample of RGB stars in NGC 7492 for Na I and O I represent real star-to-star abundance variations, while no other element shows definite evidence for such variations from this simple analysis.

\subsection{Correlated Abundance Variations of the Light Elements}

$\mathrm{C}, \mathrm{N}, \mathrm{O}, \mathrm{Na}, \mathrm{Mg}$, and $\mathrm{Al}$ are known to show correlated abundance variations from star to star among the most luminous stars in globular clusters (see, e.g., the review of Kraft 1994). Our simple spread ratio analysis (see $\S 2.4$ ) shows definite starto-star variations in abundance of both $\mathrm{O}$ and $\mathrm{Na}$ in our small sample of RGB stars in NGC 7492. Variations in Mg, if present, are smaller and subtle.

It is well established that $\mathrm{O}$ and $\mathrm{Na}$ are anticorrelated among luminous giants in globular clusters (see, e.g., Kraft 1994). Furthermore, Ramírez \& Cohen (2002) compiled the data from the literature, combined it with their own, and showed that the same linear relation can be used to fit the $\mathrm{O}$ and $\mathrm{Na}$ data for all globular clusters studied in detail thus far. The latest addition to the clusters studied in detail, NGC 2808 (Carretta et al. 2004a), does so as well.

Figure 8 shows the relationship between $\mathrm{Na}$ and $\mathrm{O}$ abundances (both with respect to Fe) for our sample in NGC 7492. Also superposed is the line representing the fit for this anticorrelation determined by Sneden et al. (2004) for the luminous giants in M3, shifted by -0.07 dex and +0.1 dex in the vertical and horizontal axis as compared to the relation we found for M13 (Cohen \& Melendez 2005). The first and last quartiles of the O-Na anticorrelation seen by Sneden et al. (2004) in their sample of luminous giants in M3 are indicated. There is a reasonably clear anticorrelation, which corresponds well with that
TABLE 7

Comparison of Abundance Ratios in NGC 7492 to Those in M3 and M13

\begin{tabular}{|c|c|c|c|c|}
\hline Species & $\begin{array}{c}\text { Mean Abundance } \\
\text { NGC } 7492,[\mathrm{X} / \mathrm{Fe}] \\
(\mathrm{dex})\end{array}$ & $\begin{array}{c}\sigma \\
(\operatorname{dex})\end{array}$ & $\begin{array}{c}\Delta[\mathrm{X} / \mathrm{Fe}] \\
(\mathrm{NGC} 7492-\mathrm{M} 3 / \mathrm{M} 13) \\
(\mathrm{dex})\end{array}$ & $\begin{array}{l}{[\mathrm{X} / \mathrm{Fe}]} \\
\text { Correction } \\
\quad(\mathrm{dex})\end{array}$ \\
\hline O............... & 0.46 & 0.15 & 0.02 & $\ldots$ \\
\hline $\mathrm{Na}$ & -0.02 & 0.21 & -0.10 & 0.00 \\
\hline $\mathrm{Mg} \ldots \ldots . .$. & 0.55 & 0.09 & 0.12 & 0.05 \\
\hline $\mathrm{Si} \ldots \ldots . . . .$. & 0.39 & 0.06 & 0.23 & 0.08 \\
\hline $\mathrm{Ca}$ & 0.08 & 0.03 & -0.09 & 0.07 \\
\hline Sc............... & 0.14 & 0.05 & 0.14 & 0.07 \\
\hline $\mathrm{Ti}$............ & 0.24 & 0.06 & 0.08 & 0.07 \\
\hline V ................ & -0.07 & & 0.01 & $\ldots$ \\
\hline Cr................... & -0.25 & 0.06 & -0.22 & -0.10 \\
\hline Mn ......... & -0.39 & 0.10 & -0.04 & -0.05 \\
\hline Co............... & 0.20 & 0.11 & 0.21 & 0.07 \\
\hline $\mathrm{Ni}$ & -0.08 & 0.01 & -0.01 & 0.00 \\
\hline $\mathrm{Cu} \ldots \ldots \ldots . . .$. & -0.64 & 0.06 & -0.02 & $\ldots$ \\
\hline $\mathrm{Zn}$ & -0.03 & 0.11 & 0.02 & $\ldots$ \\
\hline Y & -0.27 & 0.11 & -0.04 & $\ldots$ \\
\hline $\mathrm{Zr}$ & 0.36 & $\ldots$ & 0.40 & $\ldots$ \\
\hline Ва............. & 0.28 & 0.019 & 0.05 & -0.04 \\
\hline $\mathrm{La}$ & 0.12 & $\ldots$ & 0.03 & $\ldots$ \\
\hline $\mathrm{Nd} . . . \ldots . . . .$. & 0.32 & 0.11 & 0.10 & $\ldots$ \\
\hline Eu .......... & 0.61 & $\ldots$ & 0.07 & 0.00 \\
\hline
\end{tabular}

a Approximate correction to be added to the mean $[\mathrm{X} / \mathrm{Fe}]$ in $\mathrm{M} 3 / \mathrm{M} 13$ to take into account trends of $[\mathrm{X} / \mathrm{Fe}]$ with $[\mathrm{Fe} / \mathrm{H}]$ given the different metallicities of the clusters, i.e., it must be subtracted from $\Delta[\mathrm{X} / \mathrm{Fe}]$ to obtain $\Delta$ (cor) $[\mathrm{X} / \mathrm{Fe}]$.

seen for luminous giants in other well-studied Galactic GCs (see, e.g., the compilation of Cohen \& Melendez 2005). A similar correlation is detected in the outer halo GC NGC 7006 by Kraft et al. (1998).

There is marginally statistically significant evidence for a correlation between $[\mathrm{Na} / \mathrm{Fe}]$ and $[\mathrm{Mg} / \mathrm{Fe}]$ for our small sample in NGC 7492, similar to that shown in other GCs (see, for example, Figs. 12 and 13 of Sneden et al. 2004). The larger uncertainty in our deduced $[\mathrm{Mg} / \mathrm{Fe}]$ ratios makes this result quite uncertain.

\section{COMPARISON WITH THE INNER HALO GCs M3 AND M13}

We now turn to what we can learn about the chemical history of the Galaxy by comparing the abundance ratios in NGC 7492, at a galactocentric distance of $25 \mathrm{kpc}$, with those from our recent analysis of the inner halo GCs M3 and M13 (Cohen \& Melendez 2005), with $R_{\mathrm{GC}}$ of 12 and $9 \mathrm{kpc}$, respectively. We note that the atomic parameters, the analysis procedures, and the software packages used are identical in both of these studies. Hence, we should be able to detect small differences in the relative values of the abundance ratios of these three GCs.

Table 7 gives the parameter $\Delta[\mathrm{X} / \mathrm{Fe}]$, which is, for each species with detected lines, the mean abundance ratio $[\mathrm{X} / \mathrm{Fe}]$ for NGC 7492 with the average of the same parameter for M3 and M13 subtracted. Because of the large star-to-star differences in $\mathrm{O} / \mathrm{Fe}$ seen in $\mathrm{M} 3$ and especially in M13, we subtract the mean $[\mathrm{O} / \mathrm{Fe}]$ for stars in $\mathrm{M} 13$ of luminosities comparable to those we observe in NGC 7492. Similarly, Cohen \& Melendez (2005) found a luminosity dependence of $[\mathrm{Mg} / \mathrm{Fe}]$ in $\mathrm{M} 13$, most probably due to the luminosity dependence of non-LTE corrections, which were ignored. Again, in this case we subtract the mean $[\mathrm{Mg} / \mathrm{Fe}]$ of stars of comparable luminosity in M13 to those observed in NGC 7492. 


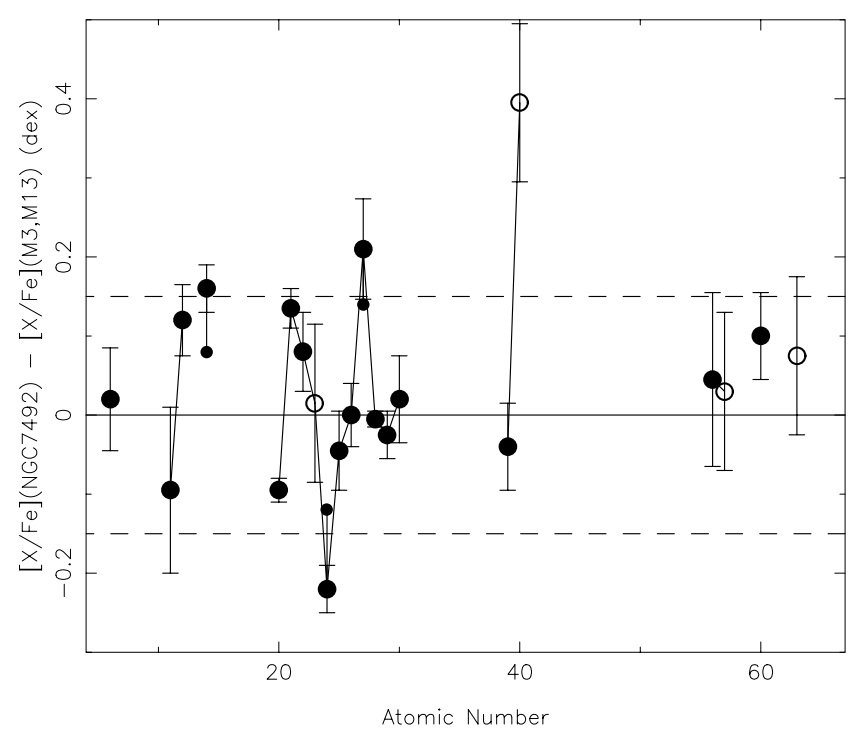

FIG. 9.- Abundance ratios $[\mathrm{X} / \mathrm{Fe}]$ for $\mathrm{NGC} 7492$, with the mean $[\mathrm{X} / \mathrm{Fe}]$ for M3 and M13 subtracted, shown as a function of atomic number. Open circles denote elements that have been detected in only one star in the NGC 7492 HIRES sample. Lines connect the points where consecutive atomic numbers have been detected. The dashed horizontal lines indicate the tolerance of \pm 0.15 dex about equality. Small filled circles indicate the results for $\mathrm{Si}, \mathrm{Cr}$, and $\mathrm{Co}$ after corrections for global trends in abundance ratio with metallicity have been applied.

If we allow \pm 0.15 dex as a tolerable range given the potential internal and systematic errors in the analyses of these three GCs, we find that $80 \%$ of the 20 elements in common have a difference of $0.0 \pm 0.15 \mathrm{dex}$, with only $\mathrm{Si}, \mathrm{Cr}$, and $\mathrm{Co}^{7}$ outside that range. Figure 9 shows the resulting differences in $[\mathrm{X} / \mathrm{Fe}]$ as a function of atomic number. The abundance ratios of $[\mathrm{X} / \mathrm{Fe}]$ for Si and Co are each larger in NGC 7492 than they are in M3 and M13, while that of $\mathrm{Cr}$ is smaller. The largest magnitude of the set of $\Delta[\mathrm{X} / \mathrm{Fe}]$ occurs for $\mathrm{Si}$, and is +0.23 dex.

Galactic chemical evolution produces trends in abundance ratios as a function of metallicity. The case of $[\mathrm{Si} / \mathrm{Fe}]$ is illustrated in Figure 10 , where metallicity is parameterized by $[\mathrm{Fe} / \mathrm{H}]$; examples for the elements $\mathrm{Ca}$, Ti, and $\mathrm{Ba}$ are given in Figures 21, 22, and 23 of Cohen \& Melendez (2005). We use the same set of high-precision analyses of GCs as in Cohen \& Melendez (2005), specifically NGC 6528 (Carretta et al. 2001), NGC 6553 (Cohen et al. 1999; Carretta et al. 2001), 47 Tuc (Carretta et al. 2004; James et al. 2004a), M71 (Ramírez \& Cohen 2002, 2003), M5 (Ramírez \& Cohen 2003), NGC 288 (Shetrone \& Keane 2000), NGC 362 (Shetrone \& Keane 2000), NGC 6752 (James et al. 2004b), M3 (Sneden et al. 2004; Cohen \& Melendez 2005), M13 (Sneden et al. 2004; Cohen \& Melendez 2005), NGC 6397 (Thévenin et al. 2001; Gratton et al. 2001; James et al. 2004a), and M15 (Sneden et al. 1997), adding in NGC 7492 as well. To characterize the behavior of the metal-poor halo field stars, we adopt abundance ratios from the recent large surveys of Gratton \& Sneden (1991), McWilliam et al. (1995), Fulbright (2000), and Johnson (2002). No effort has been made to homogenize these analyses, but since the field star surveys were carried out over the course of more than a decade, we have corrected for the difference in the solar $\mathrm{Fe}$ abundance adopted by each.

Given that NGC 7492 has $[\mathrm{Fe} / \mathrm{H}] 0.35$ dex smaller than the mean value for M3 and M13, we have attempted to evaluate the

\footnotetext{
${ }^{7}$ We ignore $\mathrm{Zr}$, as there are only a few weak lines detected the HIRES spectrum of the coolest star in our sample in NGC 7492.
}

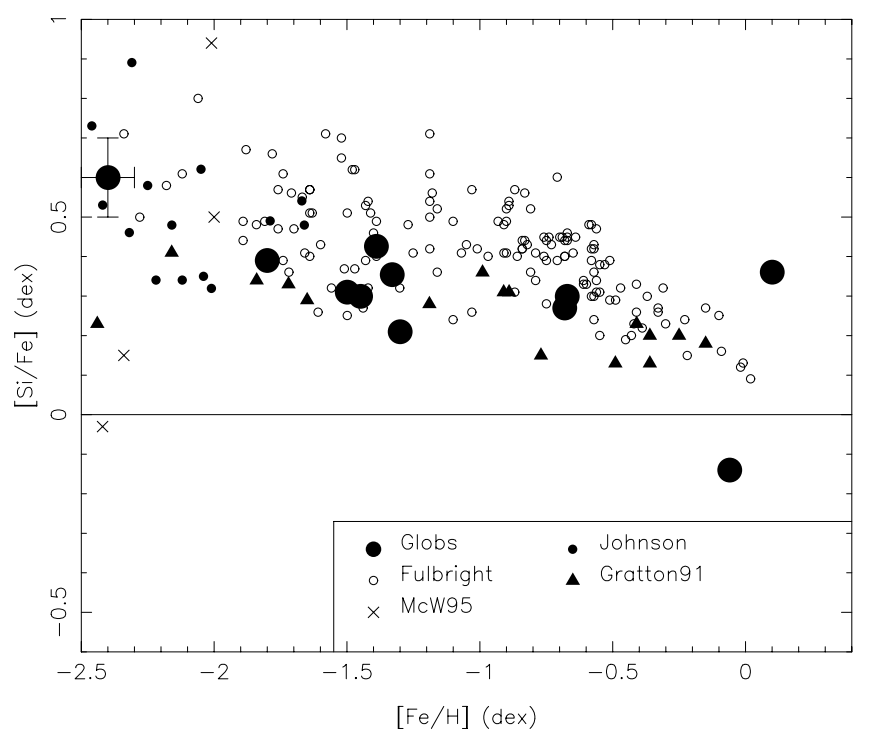

FIG. 10.-Abundance ratio $[\mathrm{Si} / \mathrm{Fe}]$, shown as a function of $[\mathrm{Fe} / \mathrm{H}]$ for a sample of 13 Galactic GCs (see text for references), indicated by large filled circles. This is compared to the same relationship for halo field stars from surveys by Fulbright (2000), McWilliam et al. (1995), Johnson (2002), and Gratton \& Sneden (1991). An error bar typical of the GCs is shown for the lowest metallicity GC.

correction to the difference caused by these global trends. We can only do this for about half of the elements studied here. This correction is given in the last column of Table 7, and is to be subtracted from the value of $\Delta[\mathrm{X} / \mathrm{Fe}]$ to form $\Delta(\operatorname{cor})[\mathrm{X} / \mathrm{Fe}]$. These corrections, which do not exceed $0.1 \mathrm{dex}$ in magnitude, bring $\Delta$ (cor) $[\mathrm{Si} / \mathrm{Fe}], \Delta$ (cor) $[\mathrm{Cr} / \mathrm{Fe}]$, and $\Delta(\mathrm{cor})[\mathrm{Co} / \mathrm{Fe}]$ within the range consistent with no difference $(0.0 \pm 0.15 \mathrm{dex})$, while not causing any additional elements to exceed the allowed range for equality. The corrections for $\mathrm{Si}, \mathrm{Cr}$, and $\mathrm{Co}$ are shown on Figure 9 as well.

Thus, after implementing the corrections for global chemical evolution, all the elements in common show identical abundance ratios [X/Fe] for NGC 7492 as for M3 and M13, allowing for a \pm 0.15 dex tolerance; $75 \%$ of the 20 values of $\Delta$ (cor) $[\mathrm{X} / \mathrm{Fe}]$ lie within the range -0.10 to +0.10 dex. This suggests that the Galactic chemical evolution of the outer halo at $R_{\mathrm{GC}} 25 \mathrm{kpc}$ has been identical to that of the well-studied inner halo GCs, at least up to the time of the formation of the old globular clusters NGC 7492, M3, and M13. In particular, our limited evidence, based on $\mathrm{Ba}, \mathrm{La}$, and $\mathrm{Eu}$ abundance ratios, suggests the neutroncapture processes, both $r$ and $s$, appear to have had similar histories throughout the spatial extent of the halo for old GC stars.

\section{SUMMARY}

We have carried out a detailed abundance analysis for 21 elements in a sample of four RGB stars in the metal-poor distant outer halo globular cluster NGC $7492([\mathrm{Fe} / \mathrm{H}]-1.80 \mathrm{dex})$. The analyzed spectra, obtained with HIRES at the Keck Observatory, are of high dispersion $(R=\lambda / \Delta \lambda=35,000)$. Most elements show no sign of star-to-star variation within our limited sample. We have, however, detected an anticorrelation between $\mathrm{O}$ and $\mathrm{Na}$ abundances similar to that seen in our previous analyses of inner halo GCs as well as in studies of relatively nearby GCs by others. A correlation between $\mathrm{Mg}$ and $\mathrm{Na}$ abundance may also be present.

We have compared the abundance ratios in NGC 7492 with those we previously determined for the much closer old halo 
GCs M3 and M13 (Cohen \& Melendez 2005), hoping that since all these analyses were carried out by the same two people within a time span of only a few months in a completely consistent manner, with the same line lists, the same atomic parameters, the same analysis codes and procedures, etc., that small differences in the abundance ratios might be detectable. We evaluate the trends of abundance ratio with metallicity for old halo stars from our data combined with published large surveys of halo field star abundances. We then apply corrections to the abundances we derived for M3 and M13 for each species, when feasible, to extrapolate them to the 0.35 dex smaller $[\mathrm{Fe} / \mathrm{H}]$ of NGC 7492 . After making such corrections, all the elements in common show identical abundance ratios for NGC 7492 and for M3 and M13, allowing for a \pm 0.15 dex tolerance, and $75 \%$ of them are then within the tolerance of \pm 0.10 dex. This suggests that the Galactic chemical evolution of the outer halo at $R_{\mathrm{GC}} 25 \mathrm{kpc}$ has been identical to that of the well-studied much closer inner halo GCs, at least up to the time of the formation of the old globular clusters NGC 7492, M3, and M13. In particular, our limited evidence, based on Ba, La, and Eu abundance ratios, suggests the neutron-capture processes, both $r$ and $s$, appear to have had similar histories throughout the spatial extent of the halo for old GC stars as well.
The presence of the $\mathrm{O} / \mathrm{Na}$ anticorrelation in NGC 7492, with $R_{\mathrm{GC}}$ of $25 \mathrm{kpc}$, and the similarity of its chemical history to that of the well-studied nearby GCs, provide new constraints on any model of GC formation in the Galactic halo.

The entire Keck/HIRES user communities owes a huge debt to Jerry Nelson, Gerry Smith, Steve Vogt, and many other people who have worked to make the Keck Telescope and HIRES a reality and to operate and maintain the Keck Observatory. We are grateful to the W. M. Keck Foundation for the vision to fund the construction of the W. M. Keck Observatory. The authors wish to extend special thanks to those of Hawaiian ancestry on whose sacred mountain we are privileged to be guests. Without their generous hospitality, none of the observations presented herein would have been possible. This publication makes use of data from the Two Micron All-Sky Survey, which is a joint project of the University of Massachusetts and the Infrared Processing and Analysis Center, funded by the National Aeronautics and Space Administration and the National Science Foundation. We are grateful to the National Science Foundation for partial support under grant AST 02-05951 to J. G. C.
Barnes, S. A. 1968, AJ, 73, 579

Buonanno, R., Corsi, C. E., Ferraro, F. R., \& Fusi Pecci, F. 1987, A\&AS, 67, 327

Carretta, E., Bragaglia, A., \& Cacciari, C. 2004a, ApJ, 610, L25

Carretta, E., Cohen, J. G., Gratton, R. G., \& Behr, B. B. 2001, AJ, 122, 1469

Carretta, E., Gratton, R., Bragaglia, A., Bonifacio, P., \& Pasquini, L. 2004b, A\&A, 416, 925

Cohen, J. G. 2004, AJ, 127, 1545

Cohen, J. G., Behr, B. B., \& Briley, M. M. 2001, AJ, 122, 1420

Cohen, J. G., Frogel, J. A., Persson, S. E., \& Elias, J. H. 1981, ApJ, 249, 481

Cohen, J. G., Gratton, R. G., Behr, B. B., \& Carretta, E. 1999, ApJ, 523, 739

Cohen, J. G., \& Melendez, J. 2005, AJ, 129, 303

Côté, P. C., Richer, H. R., \& Fahlman, G. G. 1991, AJ, 102, 1358

Cuffey, J. 1961, MNRAS, 122, 363

Cutri, R. M. et al. 2003, Explanatory Supplement to the 2MASS All-Sky Data Release (Pasadena: JPL/IPAC), http:/www.ipac.caltech.edu/2mass/releases/ allsky/doc/explsup.html

Dinescu, D. I., Majewski, S. R., Girard, T. M., \& Cudworth, K. M. 2000, AJ, 120, 1892

Fulbright, J. P. 2000, AJ, 120, 1841

Gratton, R. G., Carretta, E., Eriksson, K., \& Gustafsson, B. 1999, A\&A, 350, 955

Gratton, R. G., \& Sneden, C. 1991, A\&A, 241, 501

Gratton, R. G., et al. 2001, A\&A, 369, 87

Gustafsson, B., Bell, R. A., Eriksson, K., \& Nordlund, A. 1975, A\&A, 42, 407

Hartwick, F. D. A., \& Sargent, W. L. W. 1978, ApJ, 221, 512

Houdashelt, M. L., Bell, R. A., \& Sweigart, A. V. 2000, AJ, 119, 1448

Ibata, R., Lewis, G. F., Irwin, M., Totten, E., \& Quinn, T. 2001, ApJ, 551, 294

Irwin, M. J. 1999, in IAU Symp. 192, The Stellar Content of Local Group

Galaxies, ed. P. A. Whitelock \& R. D. Cannon (San Francisco: ASP), 409

James, G., Francois, P., Bonifacio, P., Carretta, E., Gratton, R. G., \& Spite, F. 2004a, A\&A, 427, 825

James, G., et al. 2004b, A\&A, 414, 1071

Johnson, J. A. 2002, ApJS, 139, 219
Kraft, R. P. 1994, PASP, 106, 553

Kraft, R. P., Sneden, C., Smith, G. H., Shetrone, M. D., \& Fulbright, J. 1998, AJ, 115, 1500

Kurucz, R. L. 1993, Kurucz CD-ROM 13, ATLAS9 Stellar Atmosphere Programs and $2 \mathrm{~km} / \mathrm{s}$ Grid (Cambridge: $\mathrm{SAO}$ )

McWilliam, A., Preston, G. W., Sneden, C., \& Searle, L. 1995, AJ, 109, 2757

Ramírez, S. V., \& Cohen, J. G. 2002, AJ, 123, 3277

2003, AJ, 125, 224

Ramírez, S. V., Cohen, J. G., Buss, J., \& Briley, M. M. 2001, AJ, 122, 1429

Rosenberg, A., Saviane, I., Pioto, G., \& Aparicio, A. 1999, AJ, 118, 2306

Rosenberg, A., Saviane, I., Piotto , G., \& Held, E. V. 1998, A\&A, 339, 61

Rutledge, G. A., Hesser, J. E., \& Stetson, P. B. 1997a, PASP, 109, 907

Rutledge, G. A., Hesser, J. E., Stetson, P. B., Mateo, M., Simard, L., Bolte, M., Friel, E. D., \& Copin, Y. 1997b, PASP, 109, 883

Schlegel, D. J., Finkbeiner, D. P., \& Davis, M. 1998, ApJ, 500, 525

Shetrone, M., \& Keane, M. J. 2000, AJ, 119, 840

Shortridge, K. 1993, The Figaro 2.4 Manual (Epping: Anglo-Australian Obs.)

Skrutskie, M. F., et al. 1997, in The Impact of Large Scale Near-IR Sky Surveys, ed. F. Garzon et al. (Dordrecht: Kluwer), 187

Smith, H. A. 1984, ApJ, 281, 148

Sneden, C. 1973, Ph.D. thesis, Univ. of Texas

Sneden, C., Kraft, R. P., Guhathakurta, P., Peterson, R. C., \& Fulbright, J. P. 2004, AJ, 127, 2162

Sneden, C., Kraft, R. P., Shetrone, M. D., Smith, G. H., Langer, G. E., \& Prosser, C. F. 1997, AJ, 114, 1964

Takeda, Y., Zhao, G., Takada-Hidai, M., Chen, Y., Saito, Y., \& Zhang, H. W. 2003, Chinese J. Astron. Astrophys., 3, 316

Thevenin, F., Carbonnel, C., de Freitas Pacheco, J. A., Idiart, T. P., Jasniewsicz, G., de Lavery, P., \& Plez, B. 2001, A\&A, 373, 905

Vogt, S. E., et al. 1994, Proc. SPIE, 2198, 362

Yi, S., Demarque, P., Kim, Y.-C., Lee, Y.-W., Ree, C. Lejeune, Th., \& Barnes, S. 2001, ApJS, 136, 417

Zinn, R., \& West, M. J. 1984, ApJS, 55, 45 